\title{
Análise do Uso da Cultura Maker em Contextos Educacionais: Revisão Sistemática da Literatura
}

\author{
Bruna Braga de Paula - Unifesp - paula.bruna@ unifesp.br \\ Tiago de Oliveira - Unifesp - tiago.oliveira@unifesp.br \\ Camila Bertini Martins - Unifesp - cb.martins@ unifesp.br
}

Resumo: A cultura maker tem como principal objetivo a mão na massa, possibilitar a invenção e a solução de problemas; onde criar, modificar ou construir algum objeto é o foco. No contexto educacional a cultura maker busca explorar as atividades práticas em sala de aula, possibilitando a criação e resolução de problemas ao se construir protótipos e outros objetos que visam desenvolver diferentes habilidades. Dentro deste contexto, o objetivo do trabalho é apresentar uma visão geral dos estudos que abordam a cultura maker no meio educacional, utilizando uma Revisão Sistemática da Literatura (RSL). Para a obtenção dos estudos da RSL, foram utilizadas as bases de dados da ACM Digital Library, Emerald Insight, IEEE Xplore e Springer Link. De acordo com os resultados obtidos, verifica-se que o tema tem atraído a atenção da comunidade científica internacional, sendo a maior parte das pesquisas voltadas para o domínio de aprendizagem de Ciência e Tecnologia. A RSL aponta, também, para a necessidade de mais estudos relacionados à aplicação maker no ensino, o que propiciaria uma exploração e desenvolvimento de novas ferramentas e materiais, bem como a carência de novos instrumentos para medir a sua eficácia na educação.

Palavras-chave: cultura maker, mão na massa, faça você mesmo, educação, ensino.

\section{Analysis of the Use of Maker Culture in Educational Contexts: Systematic Literature Review}

\begin{abstract}
Culture maker has as its main objective the hands-on, enabling invention and problem solving, where creating, modifying or building any object is the focus. In the educational context, the maker culture seeks to explore practical classroom activities, enabling the creation and resolution of problems by building prototypes and other objects that aim to develop different skills. Within this context, the objective of this paper is to present an overview of studies that address the maker culture in the educational environment using a Systematic Literature Review (SLR). To obtain SLR studies, we used the ACM Digital Library, Emerald Insight, IEEE Xplore and Springer Link databases. According to the results obtained, it appears that the theme has attracted the attention of the international scientific community, most of them focused on the domain of learning science and technology. The SLR also points to the need for further studies related to the maker application in teaching, which would allow the exploration and development of new tools and materials, as well as the lack of new instruments to measure their effectiveness in education.
\end{abstract}

Keywords: maker culture, hands-on, DIY, education, teaching.

\section{Introdução}

Nas escolas os alunos devem ser protagonistas do seu próprio aprendizado, utilizando ferramentas digitais com o propósito de ter um rico espaço de aprendizagem, capaz de instigar a curiosidade e propiciar momentos de reflexão acerca do mundo em 
que vivem. O intuito é que o ambiente físico da sala de aula também seja interessante para os alunos, possibilitando múltiplas interações com o universo midiático e apresentando a tecnologia como um instrumento que colabora no processo de aprendizagem (Moran, 2010).

A cultura maker, ação de colocar a mão na massa, está associada com práticas presentes na Ciência da Computação. Segundo Milne et al. (2014), as ações de um indivíduo "maker" são motivadas pela realização de projetos que reúnem computação, tecnologia e conhecimento interdisciplinar. Este movimento propôs nos últimos anos promover e estimular a criação, investigação, resoluções de problemas e autonomia; motivando o aluno a pesquisar e ir além do conteúdo que está sendo explorado em sala de aula. É um espaço em que o estudante é o principal protagonista de seu aprendizado, mesclando robótica, programação e tecnologias digitais com costura, marcenaria, materiais recicláveis e mão na massa. Assim, de forma atrativa e lúdica pode-se desenvolver a criatividade, o pensamento crítico e o trabalho em equipe.

O movimento maker está relacionado à prática na qual o aluno é protagonista do processo de construção do seu conhecimento, explorando assuntos de seu interesse e satisfação. Nessa prática ocorre a valorização da experiência do educando, permitindo que ele aprenda com seus erros e acertos, com a satisfação em compreender assuntos e temas do seu próprio interesse que estão relacionados com seu cotidiano (Blikstein, 2013). O aluno passa a atuar como autor do próprio ambiente e de forma descentralizada passa a produzir seu próprio material didático, ajudando os colegas na resolução dos problemas apresentados. Ideias que estão sendo relacionadas ao termo Pensamento Computacional que proporcionam um aprendizado criativo, no qual o modelo de troca de conhecimento entre mediador e estudante deixa de ser somente expositivo (Blikstein et al., 2012). Considerando a sua importância e com base nas considerações dos espaços e materiais necessários para aprender a fazer, foi realizada uma Revisão Sistemática da Literatura (RSL), com o objetivo de construir uma visão geral dos estudos que tratam de cultura maker no mundo, visando a sua aplicabilidade no ensino; tendo em vista que, embora não tenha sido inicialmente a proposta para ser aplicada em sala de aula, a cultura maker vem sendo utilizada por professores no ambiente acadêmico e escolar devido às suas contribuições nos processos de ensino e de aprendizagem.

Este artigo está organizado em quatro seções, sendo que na seção 2 é apresentada a Metodologia de Pesquisa, na seção 3 os Resultados obtidos e na seção 4, as Considerações Finais.

\section{Metodologia de Pesquisa}

Para executar a RSL foram utilizados as diretrizes e o modelo de protocolo proposto por Kitchenham et al. (2007). Nesta seção, são definidas as questões de pesquisa, a String de busca e as técnicas que serão usadas para avaliar os artigos e realizar a extração dos dados.

\subsection{Questões de Pesquisa}

Como o objetivo desta RSL é fornecer um panorama dos estudos apresentados na literatura envolvendo o uso e a aplicabilidade da cultura maker nos ambientes educacionais, definiu-se quatro questões de pesquisa principais $(\mathrm{QP})$ que, em conjunto, buscam atender ao objetivo proposto.

As questões formuladas são: QP1: Como é a distribuição e aplicabilidade do movimento maker nos ambientes educacionais? QP2: Quais são os recursos/ferramentas/ infraestrutura utilizados na concepção dos laboratórios? QP3: Quais são as estratégias e métodos que têm sido adotados para medir a eficácia do aprendizado nessa abordagem e 
contexto educacional? QP4: Quais são as principais vantagens, desvantagens e problemas que vem sendo reportados na literatura ao adotar essa abordagem?

\subsection{Estratégia de busca}

A estratégia de busca utilizada é composta por duas etapas: na primeira definemse as palavras-chave e a semântica da pesquisa; na segunda, por sua vez, as bibliotecas digitais, revistas e conferências que serão utilizadas como fonte de estudos (Kitchenham et al., 2007). As palavras-chave (e seus sinônimos) definidas por meio das questões de pesquisa foram:

- Maker culture (movement maker, maker movement, maker activity, maker activities, space maker, makerspace, maker space, do it yourself, fab lab, fablab, fabrication laboratory, fabrication laboratories);

- Education (teaching, learning).

A partir disso, construiu-se a String de busca, por meio da combinação das palavras-chave e de seus sinônimos, usando operadores lógicos (OU) entre os sinônimos identificados e o operador (E) entre as palavras-chave. Como resultado, a seguinte String de busca foi utilizada na RSL:

String = ("maker culture" OR "movement maker" OR "maker movement" OR "maker activity" OR "maker activities" OR "space maker" OR "makerspace" OR "maker space" OR "do it yourself" OR "fab lab" OR "fablab" OR "fabrication laboratory" OR "fabrication laboratories") AND ("education" OR "teaching" OR "learning")

Para a segunda etapa, após a definição do problema e a construção da String de busca, as bases bibliográficas utilizadas para a obtenção dos estudos foram:

- ACM Digital Library (http://portal.acm.org).

- Emerald Insight (https://www.emerald.com/insight/).

- IEEE Xplore (http://ieeexplore.ieee.org).

- Springer Link (http://link.springer.com).

\subsection{Seleção dos estudos}

O próximo passo da definição do protocolo da RSL, visando melhorar os resultados obtidos, consiste na criação de alguns critérios para os artigos que serão selecionados (inclusão, exclusão e qualidade) com base nas questões de pesquisa, String de busca e bases bibliográficas definidas (Kitchenham et al., 2007).

Um estudo deve ser incluído na RSL baseado em sua relevância com relação às questões de pesquisa propostas. Sendo assim, apenas serão incluídos artigos que descrevam pesquisas relacionadas ao tema abordado. Os critérios de inclusão e exclusão, apresentados no Quadro 1, devem ser aplicados aos 1.193 estudos provenientes das buscas.

Quadro 1 - Critérios de inclusão e exclusão na RSL.

\section{Critérios de Inclusão}

- Revisados por especialistas que fornecem respostas paras as perguntas de pesquisa;

- Apresentam alguma abordagem maker nos ambientes educacionais e utilização dos laboratórios (espaços e infraestrutura);

- Fornecem alguma evidência empírica sobre as vantagens/ desvantagens/ problemas da utilização da cultura maker no ensino;

- Abrangem todos os anos até os dias atuais;

\section{Critérios de Exclusão}

- Estudos não completos e que apresentam lacunas no trabalho e/ou não apresentam fundamentação teórica adequada;

- Estudos publicados como "short-papers" e ou estudos secundários, como outras revisões sistemáticas, surveys e capítulos de livros;

- Artigos similares (quando dois ou mais artigos tem conteúdo muito parecido será mantido apenas o estudo mais recente);

- Estudos que não foram revisados por especialistas (peer review);

V. $17 \mathrm{~N}^{\mathrm{o}}$ 3, dezembro, 2019

DOI: 
- Relatem alguma abordagem maker no ensino, independente da aplicabilidade e metodologia utilizada;

- Tratam de materiais e infraestrutura dos laboratórios makers com foco educacional;

Por fim, os critérios de qualidade ajudam a selecionar e identificar os principais estudos em relação às suas qualidades e seu potencial em relação às questões de pesquisa. A análise da qualidade do estudo aumenta a precisão dos resultados da extração de dados, de modo a obter estudos relevantes e capazes de abordar cada questão de pesquisa. Para isso, uma série de questões de avaliação de qualidade foram formuladas para avaliar a credibilidade, integridade e relevância dos estudos selecionados, sendo apresentadas na Tabela 1. Cada questão formulada permitia apenas três respostas opcionais com as seguintes pontuações: "Sim" = 1,0, "Parcialmente" = 0,5 ou "Não" = 0 .

Os estudos que apresentam uma taxa de qualidade de no mínimo $50 \%$ serão incluídos na RSL, tendo sido sumarizados na Tabela 2. A taxa de qualidade para cada artigo é a média aritmética obtida pelas respostas atribuídas a cada uma das sete questões.

Tabela 1 - Resultado da análise do critério de qualidade dos artigos selecionados na RSL.

\begin{tabular}{|c|c|c|c|}
\hline Questões & Sim & Parcialmente & Não \\
\hline A abordagem apresentada é claramente explicada? & $27(23,5 \%)$ & $5(4,3 \%)$ & $83(72,2 \%)$ \\
\hline Existe uma descrição clara do nível educacional? & $27(23,5 \%)$ & $3(2,6 \%)$ & $85(73,9 \%)$ \\
\hline $\begin{array}{l}\text { Existe uma descrição adequada das estratégias } \\
\text { adotadas para explorar a cultura maker? }\end{array}$ & $7(6,1 \%)$ & $4(3,5 \%)$ & $104(90,4 \%)$ \\
\hline Existe uma descrição dos resultados do estudo? & $3(2,6 \%)$ & $24(20,9 \%)$ & $88(76,5 \%)$ \\
\hline $\begin{array}{l}\text { Existe uma descrição adequada do método de } \\
\text { pesquisa? }\end{array}$ & $27(23,5 \%)$ & $5(4,3 \%)$ & $83(72,2 \%)$ \\
\hline O estudo foi avaliado empiricamente? & $18(15,7 \%)$ & $12(10,4 \%)$ & $85(73,9 \%)$ \\
\hline $\begin{array}{l}\text { O estudo utilizou ou avaliou alguma ferramenta } \\
\text { tecnológica? }\end{array}$ & $26(22,6 \%)$ & $1(0,9 \%)$ & $88(76,5 \%)$ \\
\hline
\end{tabular}

Vale ressaltar que o critério de qualidade definido está relacionado à aderência do artigo ao escopo da RSL. Isso não quer dizer que o artigo, caso não atinja a pontuação mínima tenha qualidade técnica ou mérito inferior aos demais estudos, significando apenas que o artigo não traz informações relevantes suficientes para a RSL realizada.

Tabela 2 - Artigos incluídos na RSL e suas pontuações da avaliação do critério de qualidade.

\begin{tabular}{ccc|ccc}
\hline ID & Autor & \% & ID & Autor & \% \\
\hline $\mathbf{1}$ & (Irie et al., 2018) & $86 \%$ & $\mathbf{1 7}$ & (Chen; Lin, 2019) & $79 \%$ \\
$\mathbf{2}$ & (Youmans et al., 2018) & $50 \%$ & $\mathbf{1 8}$ & (Hira et al., 2014) & $64 \%$ \\
$\mathbf{3}$ & (Ferretti, 2019) & $57 \%$ & $\mathbf{1 9}$ & (Yeh et al., 2018) & $79 \%$ \\
$\mathbf{4}$ & (Bensenouci; Brahimi, 2017) & $50 \%$ & $\mathbf{2 0}$ & (Putra et al., 2018) & $64 \%$ \\
$\mathbf{5}$ & (Olga; Tatiana, 2018) & $64 \%$ & $\mathbf{2 1}$ & (Beavers et al., 2019) & $64 \%$ \\
$\mathbf{6}$ & (Kaar; Stary, 2019) & $57 \%$ & $\mathbf{2 2}$ & (Shaw; Wendell, 2016) & $64 \%$ \\
$\mathbf{7}$ & (Rosenfeld et al., 2019) & $64 \%$ & $\mathbf{2 3}$ & (Chytas et al., 2018) & $79 \%$ \\
$\mathbf{8}$ & (Vargas et al., 2018) & $50 \%$ & $\mathbf{2 4}$ & (Gadjanski et al., 2016) & $64 \%$ \\
$\mathbf{9}$ & (Nemorin, 2016) & $64 \%$ & $\mathbf{2 5}$ & (Lensing et al., 2018) & $79 \%$ \\
$\mathbf{1 0}$ & (Chen; Wu, 2017) & $57 \%$ & $\mathbf{2 6}$ & (Fasso; Knight, 2019) & $64 \%$ \\
$\mathbf{1 1}$ & (Braga; Guttmann, 2019) & $79 \%$ & $\mathbf{2 7}$ & (Hynes et al., 2017) & $50 \%$ \\
$\mathbf{1 2}$ & (Sang; Simpson, 2019) & $57 \%$ & $\mathbf{2 8}$ & (Chytas; Tsilingiris, 2019) & $57 \%$ \\
$\mathbf{1 3}$ & (Zhang et al., 2018) & $64 \%$ & $\mathbf{2 9}$ & (Hsu et al. 2017) & $50 \%$ \\
$\mathbf{1 4}$ & (Bairaktarova; Johri, 2016) & $64 \%$ & $\mathbf{3 0}$ & (Trust et al., 2017) & $86 \%$ \\
$\mathbf{1 5}$ & (Tan, 2018) & $64 \%$ & $\mathbf{3 1}$ & (Khalifa; Brahimi, 2017) & $64 \%$ \\
$\mathbf{1 6}$ & (Brown, 2015) & $57 \%$ & & & \\
\hline
\end{tabular}




\subsection{Extração dos dados}

Após as definições da pesquisa e dos processos de seleção, pode-se obter conclusões sobre as questões de pesquisa por meio da análise de dados. O processo de extração de dados deve ser realizado por meio da leitura do texto completo de cada um dos trabalhos selecionados nas etapas anteriores. Durante esta fase, os dados serão extraídos de cada um dos artigos incluídos nesta RSL de acordo com um formulário de extração pré-definido, ilustrado na Tabela 3. Assim, é possível registrar os detalhes completos dos artigos, além de ser mais específico sobre como cada um deles aborda as questões da pesquisa.

Tabela 3 - Formulário de extração dos dados na RSL.

\begin{tabular}{|c|c|c|}
\hline Número & Dados do estudo & Descrição \\
\hline 1 & Identificador do estudo & Identificador único para o estudo \\
\hline 2 & Data da extração & Data da extração do artigo \\
\hline 3 & Autores, ano título e país & $\begin{array}{l}\text { Identificação dos autores, ano e país de publicação do } \\
\text { artigo }\end{array}$ \\
\hline 4 & Tipo de artigo & Periódicos e Conferências \\
\hline 5 & Método de pesquisa & $\begin{array}{l}\text { Experimento, estudo de caso, survey, etnografia, análise } \\
\text { conceitual, revisão, não aplicável }\end{array}$ \\
\hline 6 & Domínios de Aprendizagem & $\begin{array}{l}\text { Bem-estar físico, social e emocional, cultura e artes, } \\
\text { alfabetização e comunicação, abordagens e aprendizagem } \\
\text { cognitiva, matemática e ciência e tecnologia. }\end{array}$ \\
\hline 7 & QP1 - Nível de educação & $\begin{array}{l}\text { Ensino Infantil, Ensino Fundamental, Ensino Médio, } \\
\text { Ensino Superior e Especializações }\end{array}$ \\
\hline 8 & QP2 - Materiais/ recursos & $\begin{array}{l}\text { Ferramentas, impressoras, cortadoras, materiais } \\
\text { recicláveis, Lego, tecnologia, Arduino }\end{array}$ \\
\hline 9 & QP2 - Infraestrutura & $\begin{array}{l}\text { Sala de aula, laboratórios, estação de trabalhos, } \\
\text { bibliotecas, não aplicável }\end{array}$ \\
\hline 10 & $\begin{array}{l}\text { QP3 - Estratégia/ Método para } \\
\text { medir eficácia }\end{array}$ & $\begin{array}{l}\text { Avaliações/ Questionário para identificar melhorias nas } \\
\text { habilidades cognitivas, físicas ou motoras, assimilação de } \\
\text { conteúdo, socialização, cooperação, autonomia e } \\
\text { comunicação }\end{array}$ \\
\hline 11 & QP4 - Evidências Vantagens & Citado/ Não citado \\
\hline 12 & $\begin{array}{l}\text { QP4 - Evidências Desvantagens/ } \\
\text { problemas }\end{array}$ & Citado/ Não citado \\
\hline
\end{tabular}

\section{Resultados}

Após as etapas descritas anteriormente, procedeu-se a obtenção dos estudos, a partir das bases bibliográficas e String de busca definidas. Este processo foi executado manualmente usando as ferramentas de busca providas por cada uma das bases e, após a realização dos critérios de inclusão e exclusão, obteve-se os resultados apresentados no fluxograma da Figura 1. Do total de 115 artigos, realizou-se a análise do critério de qualidade, como definido na subseção 2.3, tendo sido mantido na RSL, como resultado, os estudos apresentados na Tabela 2.

A primeira característica analisada foi com relação ao ano de publicação. Os 31 artigos incluídos na RSL foram publicados entre 2014 e 2019. A Figura 2 apresenta a distribuição da quantidade de artigos incluídos por ano de publicação. Podemos observar que há uma tendência de aumento nos últimos anos de publicações referentes à cultura maker com enfoque na área educacional, evidenciando que o tema tem atraído a atenção da comunidade científica internacional.

A segunda característica analisada refere-se ao tipo de artigo, sendo a maioria documentos publicados em periódicos científicos $(54,84 \%)$, enquanto o restante encontra-se em conferências $(45,16 \%)$. 


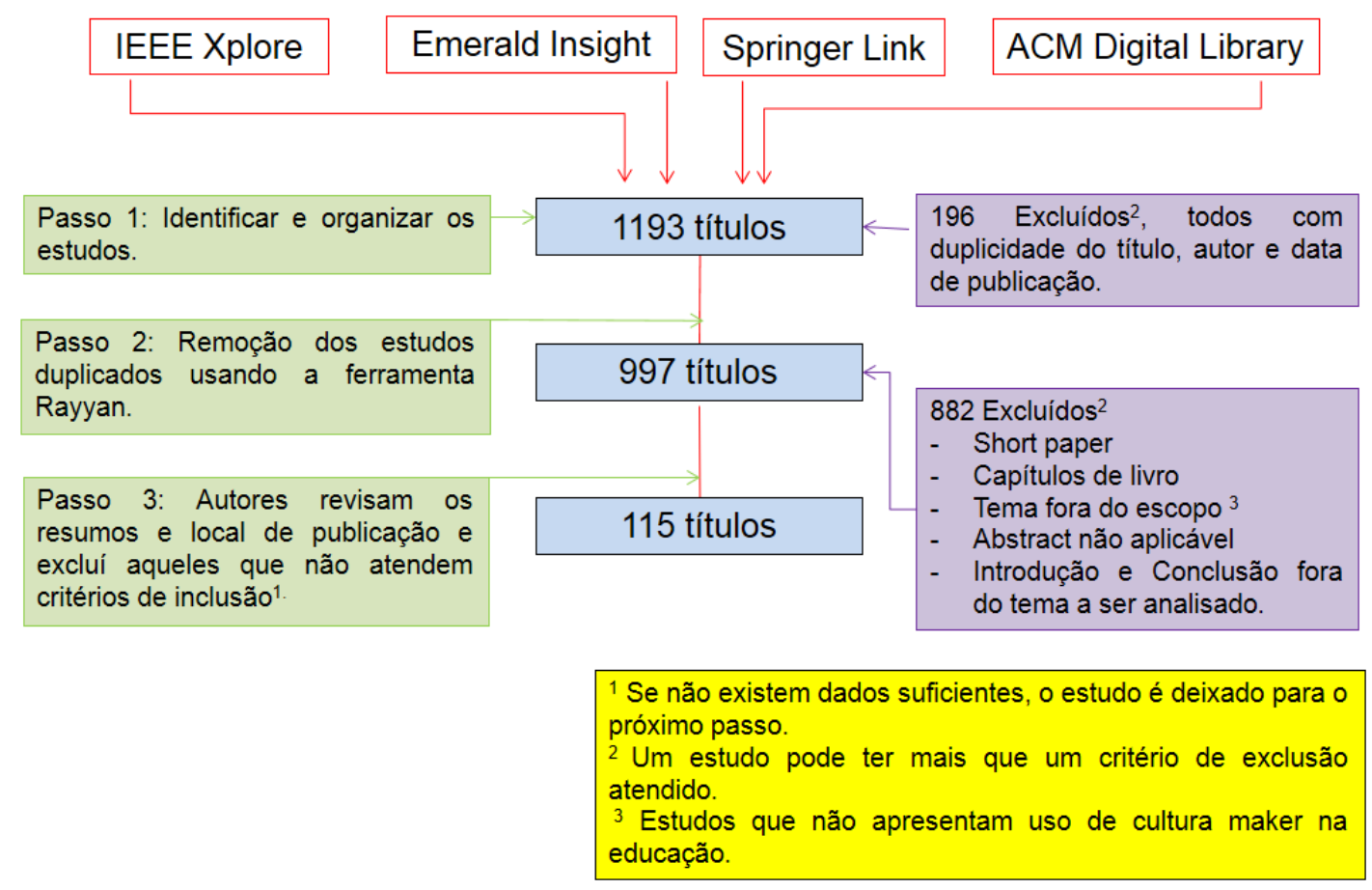

Figura 1 - Fluxograma da extração de artigos por base bibliográfica.

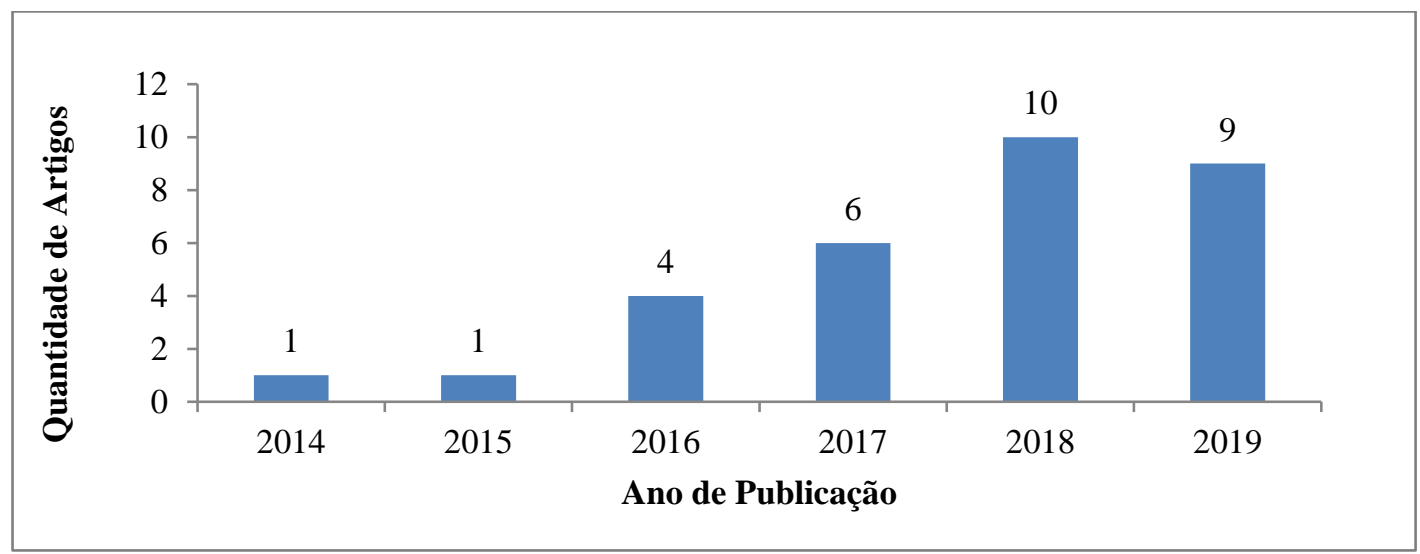

Figura 2 - Quantidade de artigos por ano de publicação.

A terceira e última característica geral analisada foi com relação aos países de publicação dos artigos de conferência e periódicos científicos. Os dados referem-se aos países que os artigos incluídos foram publicados, caso contrário, referem-se ao país referente ao primeiro autor.

Tabela 4 - Quantidade de artigos selecionados por país.

\begin{tabular}{clcl}
\hline \multicolumn{2}{c}{ Conferências } & \multicolumn{2}{c}{ Periódicos Científicos } \\
\hline $\begin{array}{c}\text { Quantidade de } \\
\text { Publicações }\end{array}$ & \multicolumn{1}{c}{ País } & $\begin{array}{c}\text { Quantidade de } \\
\text { Publicações }\end{array}$ & \multicolumn{1}{c}{ País } \\
2 & Arábia Saudita & 2 & Austrália \\
2 & Dubai & 1 & Brasil \\
2 & Espanha & 1 & China \\
1 & Indonésia & 2 & Cingapura \\
1 & Japão & 1 & Espanha \\
1 & México & 1 & Israel \\
1 & Nova Zelândia & 1 & Itália \\
3 & Estados Unidos & 1 & Rússia \\
1 & Sérvia & 7 & Estados Unidos \\
\hline
\end{tabular}

V. $17 \mathrm{~N}^{\mathrm{o}}$ 3, dezembro, 2019 
Pode-se observar, na Tabela 4, a distribuição geográfica das publicações, incluindo diversos países. Contudo, os Estados Unidos se destacaram por possuir um número de artigos relativamente maior do que os demais sendo $21,42 \%$ em conferências e $41,17 \%$ em periódicos.

Após a análise descritiva dos artigos incluídos na RSL, segue a investigação dos dados referentes às questões de pesquisa. A primeira questão de pesquisa investigada foi QP1: Como é a distribuição e aplicabilidade do movimento maker nos ambientes educacionais? Para responder essa questão relacionou-se o contexto educacional com os domínios de aprendizagem mencionados pela UNESCO (2013). Desta forma, os artigos foram analisados associando-os aos sete domínios de aprendizagem, por meio da contabilização de quais domínios cada artigo se enquadrava. A Figura 3 representa o percentual de artigos associados aos sete domínios de aprendizagem, enquanto a Figura 4 apresenta o nível educacional retratado.

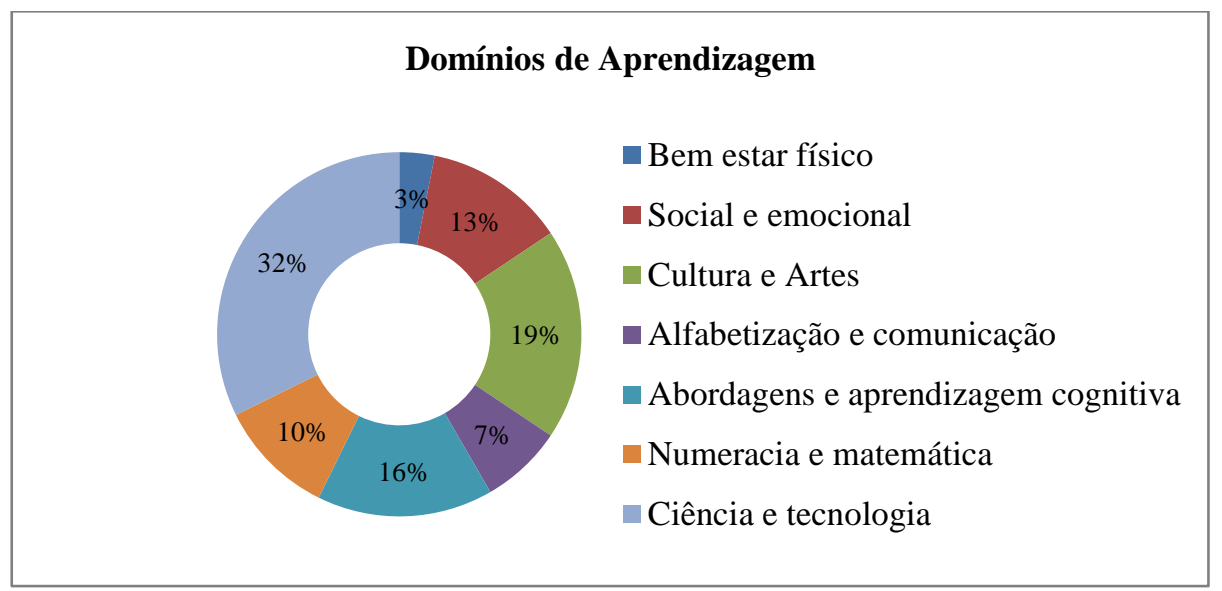

Figura 3 - Quantidade de artigos referentes ao contexto aplicado.

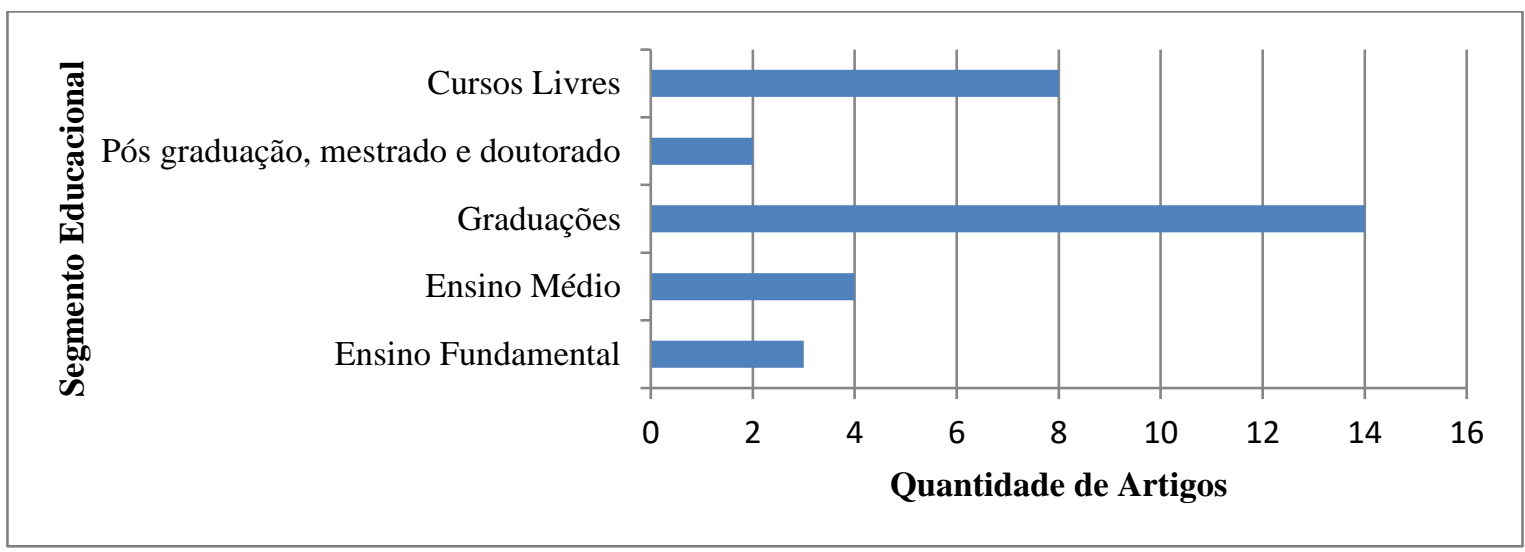

Figura 4 - Quantidade de artigos referentes ao Nível Educacional aplicado.

Observando as Figura 3 e Figura 4, verificou-se como resposta à pergunta QP1, que a aplicação da cultura maker tem sido reportada majoritariamente no ensino superior. Além disso, pode-se identificar que a maior parte dos estudos $(45 \%)$ retrata um contexto educacional relacionado ao domínio de aprendizagem de Ciência e Tecnologia e com poucos estudos no domínio de Bem-Estar Físico e de Alfabetização e Comunicação.

A segunda questão de pesquisa analisada foi QP2: Quais são os recursos/ ferramentas/ materiais/ infraestrutura utilizados na concepção dos laboratórios? Para responder essa questão foram analisados a infraestrutura e recursos/ ferramentas reportadas nos estudos para aplicação da cultura maker nos ambientes educacionais. Do 
ponto de vista do contexto da infraestrutura, têm-se que em 20 artigos (64,5\%) foi indicada utilização de algum espaço da escola ou universidade, espaços de cursos livres ou espaços emprestados/alugados, enquanto em 11 artigos (35,5\%) não foi especificada claramente a infraestrutura necessária ou utilizada para a abordagem maker. Os nove artigos reportaram o uso da cultura maker dentro da sala de aula (29\%), dois em laboratório (7\%), dois em biblioteca (7\%) e sete em estação de trabalho localizada em espaço externo a escola (22\%). Ou seja, a abordagem maker é muito utilizada em sala de aula e estações de trabalho externo.

Sobre o uso dos recursos e ferramentas citadas na pesquisa, cinco artigos indicaram o uso do Arduino com foco em explorar a linguagem de programação $\mathrm{C} / \mathrm{C}++$, 13 referiram ao uso da impressora 3D, seis mencionaram a cortadora a laser e 10 utilizavam ferramentas gerais tais como: martelo, serrote, pistola de cola quente, estilete, multímetro, chave de fenda, alicate, ferro de solda, led, motores, sensores, componentes eletrônicos, jumpers, dentre outros. Nos demais artigos da RSL não foram mencionados os recursos ou ferramentas utilizadas. A RSL evidenciou grande uso de impressoras 3D e de ferramentas gerais em conjunto com a necessidade de infraestruturas descritas anteriormente na Tabela 5, buscando-se propiciar um ambiente educacional adequado para a exploração de tecnologias, trabalho colaborativo e de socialização.

Além disso, uma questão não encontrada nos artigos refere-se à adoção de outras opções de ferramentas e materiais como, por exemplo, materiais recicláveis. O uso desse tipo de material integrado a uma abordagem maker poderia ser utilizado tanto do ponto de vista pedagógico quanto do ponto de vista de sua implantação no contexto educacional. O uso de materiais recicláveis com vistas aos processos de ensino e de aprendizagem pode permitir trabalhar conceitos bastante atuais nos temas de sustentabilidade e de meio ambiente, já que a consciência ecológica através da educação fundamentada na ética ambiental permite a construção de valores para a conservação do meio ambiente (Sirvinskas, 2002). Além disso, ao utilizar materiais recicláveis há o barateamento do custo de aquisição e implantação de um ambiente maker; auxiliando sua disseminação em diferentes setores educacionais, como em escolas públicas.

Seguindo com a análise, tem-se a terceira questão QP3: Quais são as estratégias e métodos que têm sido adotados para medir a eficácia do aprendizado nessa abordagem e contexto educacional? Com relação ao método de avaliação dos alunos, nove artigos (29\%) destacaram a importância da utilização de avaliações como medida de eficácia do desempenho dos alunos, um artigo (3\%) apontou a utilização da construção de vídeos, um artigo (3\%) o uso de questionários e 20 artigos (65\%) não mencionaram como medir o desempenho dos estudantes.

A próxima questão avaliada foi QP4: Quais são as principais vantagens, desvantagens e problemas que vem sendo reportadas na literatura ao adotar essa abordagem? Nessa questão não houve artigos que trouxessem pesquisas concretas sobre as desvantagens e problemas de explorar a cultura maker. Uma constatação interessante refere-se ao fato de não ter sido encontrado na RSL estudos sobre colaboração, entusiasmo, proatividade, motivação, criatividade, entre outras competências, habilidades ou comportamentos que poderiam ser gerados ou desenvolvidos nos alunos ao decorrer do uso da abordagem maker. A grande maioria dos artigos (90\%) menciona evidências positivas como: aprendizado centrado no aluno e autêntico, resolução de problemas, criação de protótipos, inovação, criatividade, colaboração e ludicidade. Apenas $10 \%$ dos artigos não mencionaram os benefícios do uso da cultura maker na educação, contudo, nenhum desses artigos apresentaram evidências negativas. Em relação aos problemas, as ocorrências mais citadas foram o custo de materiais e a dificuldade de montar um laboratório maker contendo ferramentas necessárias. 


\section{Considerações Finais}

De acordo com a RSL realizada, a aplicação da cultura maker na área educacional vem sendo pesquisada em vários países, com ênfase maior nos Estados Unidos. A análise dos estudos mostrou que há várias estratégias de aplicabilidade na educação e que as ferramentas ou recursos que são mais utilizados em sua exploração são a impressora 3D, ferramentas gerais e cortadoras a laser. Os artigos que tratam sobre a cultura maker não destacaram como era sua aplicabilidade no ensino e nem como acontecia essa exploração, bem como não relacionaram aos conteúdos curriculares que poderiam ser explorados pelos alunos. Identificou-se também alguns problemas e desvantagens da abordagem maker no ensino, como a dificuldade de incorporação da tecnologia no currículo, a aquisição de recursos e ferramentas necessárias, disponibilização de espaços adequados, equipamentos limitados, treinamento, suporte aos professores, crenças dos professores em relação a papéis professor-aluno, ênfases curriculares e práticas de avaliação que não são bem definidas. Também vale ressaltar que há uma carência no desenvolvimento de novos instrumentos para medir a eficácia de se explorar a cultura maker na educação, bem como a falta de estudos relacionados tanto a qualidade do aprendizado que os alunos obtiveram quanto as habilidades e competências desenvolvidas com o uso dessa abordagem.

\section{Referências Bibliográficas}

BAIRAKTAROVA, D.; JOHRI, A. The role of virtual objects in performing engineering related task. In: 2016 IEEE FRONTIERS IN EDUCATION CONFERENCE (FIE), 2016, USA. Proceedings. Institute of Electrical and Electronics Engineers, 2016, p.1-5. BENSENOUCI, A.; BRAHIMI, T. Powering makerspace wirelessly: Opportunities and challenges. In: 2017 LEARNING AND TECHNOLOGY CONFERENCE (L\&T), 2017, Saudi Arabia. Proceedings. Institute of Electrical and Electronics Engineers, 2017, p.1-5.

BLIKSTEIN, P. Digital fabrication and 'making' in education: The democratization of invention. In: WALTER-HERRMANN J.; BUCHING C. (Eds). FabLabs: Of machines, makers and inventors. Bielefeld: Transcript, 2013, p.1-22.

BLIKSTEIN, P.; COOPER, S.; KOLLER, D.; SAHAMI, M.; PIECH, C. Modeling How Students Learn to Program. In: 43RD ACM TECHNICAL SYMPOSIUM ON COMPUTER SCIENCE EDUCATION, 2012, USA. Proceeding of the 43rd ACM technical symposium on Computer Science Education, 2012, p.153-160.

BRAGA, M.; GUTTMANN, G. The Knowledge Networks in a Makerspace: The Topologies of Collaboration. International Journal of Science and Mathematics Education, Singapore, v.17, n.1, p 13-30, 2019.

BROWN, A. 3D Printing in Instructional Settings: Identifying a Curricular Hierarchy of Activities. Tech Trends, USA, v.59, n.5, p 16-24, 2015.

BEAVERS, K.; CADY, J.E.; JIANG, A.; MCCOY, L. Establishing a maker culture beyond the makerspace. Library Hi Tech, v.37, n.32, 2019.

CHEN, C.-S; LIN, J.-W. A Practical Action Research Study of the Impact of MakerCentered STEM-PjBL on a Rural Middle School in Taiwan. International Journal of Science and Mathematics Education, USA, v.17, n.1, p 85-108, 2019.

CHEN, Y.; WU C. The hot spot transformation in the research evolution of maker. Scientometrics, v.113, n.3, p 1307-1324, 2017

CHYTAS, C.; TSILINGIRIS, A. Exploring Computational Thinking Skills in 3D Printing: A Data Analysis of an Online Makerspace. In: 2019 IEEE GLOBAL ENGINEERING EDUCATION CONFERENCE (EDUCON), 2019, United Arab Emirates. Proceedings. Institute of Electrical and Electronics Engineers, 2019.

V. $17 \mathrm{~N}^{\mathrm{o}}$ 3, dezembro, 2019 RENOTE DOI: 
CHYTAS, S.; DIETHELM, I.; TSILINGIRIS, A. Learning programming through design: An analysis of parametric design projects in digital fabrication labs and an online makerspace. In: 2018 IEEE GLOBAL ENGINEERING EDUCATION CONFERENCE (EDUCON), 2018, Spain. Proceedings. Institute of Electrical and Electronics Engineers, 2018.

FASSO, W.; KNIGHT, B.A. Identity development in school makerspaces: intentional design. International Journal of Technology and Design Education, p 1-20, 2019.

FERRETTI, F. Mapping do-it-yourself Science. Life Sciences, Society and Policy, 2019. Disponível em: <https://lsspjournal.biomedcentral.com/articles/10.1186/s40504-0180090-1> Acesso em: 14 ago.2019.

GADJANSKI, I.; RADULOVIC, D.; VRANIC, F. RASPOPOVIC, M. Formation of Fab lab Petnica. In: 2016 INTERNATIONAL CONFERENCE MULTIDISCIPLINARY ENGINEERING DESIGN OPTIMIZATION (MEDO), 2016, Serbia. Proceedings. Institute of Electrical and Electronics Engineers, 2016.

HIRA, A.; JOSLYN, C. H.; HYNES, M.M. Classroom makerspaces: Identifying the opportunities and challenges. In: 2014 IEEE FRONTIERS IN EDUCATION CONFERENCE (FIE), 2014, Spain. Proceedings. Institute of Electrical and Electronics Engineers, 2014.

HSU, Y-C.; BALDWIN, S.; CHING, Y-H. Learning through Making and Maker Education. Tech Trends, v.61, n.6, p 589-594, 2017.

HYNES, M. M.; HYNES, W.J. If you build it, will they come? Student preferences for Makerspace environments in higher education. Proceedings. International Journal of Technology and Design Education, v.28, n.3, p 867-883, 2017.

IRIE, N.R.; HSU, Y-C.; CHING, Y-H. Makerspaces in Diverse Places: A Comparative Analysis of Distinctive National Discourses Surrounding the Maker Movement and Education in Four Countries. Tech Trends, v.63, n.4, p 397-407, 2018.

KAAR, C.; SATRY, C. Structuring Academic Education in Makerspaces: Consolidated Findings from the Field. In: 2019 IEEE GLOBAL ENGINEERING EDUCATION CONFERENCE (EDUCON), 2019, United Arab Emirates. Proceedings. Institute of Electrical and Electronics Engineers, 2019.

KHALIFA, S.; BRAHIMI, T. Makerspace: A novel approach to creative learning. In: 2017 LEARNING AND TECHNOLOGY CONFERENCE (L\&T), 2017, Saudi Arabia. Proceedings. Institute of Electrical and Electronics Engineers, 2017.

KITCHENHAM, B.; CHARTERS, S. Guidelines for performing systematic literature reviews in software engineering. EBSE Technical Report, 2007, 57p.

LENSING, K.; SCHWUCHOW, B.; OEHLANDT, S.; HAERTEL, T. How Makerspaces Help to Participate in Technology: Results of a Survey to Gain Data about Learners' Activities in Makerspaces. In: 2018 WORLD ENGINEERING EDUCATION FORUM - GLOBAL ENGINEERING DEANS COUNCIL (WEEF_GEDC), 2018, USA. Proceedings. Institute of Electrical and Electronics Engineers, 2018.

MILNE, A. P.; RIECKE, B. E.; ANTLE, A. N. Exploring Maker Practice: Common Attitudes, Habits and Skills from the Maker Community. Studies, v.19, n.21, 2014.

MORAN, J. M. Ensino e aprendizagem inovadores com tecnologias audiovisuais e telemáticas. In: MORAN, J. M.; MASETTO, M. T.; BEHRENS, M. A. (Ed). Novas tecnologias e mediação pedagógica. Campinas, SP: Papirus, 2010.

NEMORIN, S. The frustrations of digital fabrication: an auto/ethnographic exploration of '3D Making' in school. International Journal of Technology and Design Education, v.27, n.4, p 517-535, 2016.

OLGA, P. Z.; TATIANA, L. K. Reimagine Teacher Training for Performing in Information-Oriented Society (FabLab). In: 2018 3rd RUSSIAN-PACIFIC 
CONFERENCE ON COMPUTER TECHNOLOGY AND APPLICATIONS (RPC), Russia, 2018. Proceedings. Institute of Electrical and Electronics Engineers, 2018. PUTRA, A. B. N.R.; MUKHADIS, A.; POERWANTO, E.E.; IRDIANTO, W.; SEMBIRING, A.I. Edmodo-Based Makerspace as E-Learning Technology to Improve the Management Project of Vocational Students in the Disruptive Technology Era. In: 2018 INTERNATIONAL CONFERENCE ON SUSTAINABLE INFORMATION ENGINEERING AND TECHNOLOGY (SIET), Indonesia, 2018. Proceedings. Institute of Electrical and Electronics Engineers, 2018.

ROSENFELD, S.; YAYON, M.; HALEVI, R.; BLONDER, R. Teachers as Makers in Chemistry Education: an Exploratory Study. International Journal of Science and Mathematics Education, v.17, n.1, p 125-148, 2019.

SANG, W.; SIMPSON, A. The Maker Movement: a Global Movement for Educational Change. International Journal of Science and Mathematics Education, v.17, n.1, p 65-83, 2019.

SHAW, F.; WENDELL, K. Examining two learner approaches in a making activity with university students. In: 2016 IEEE FRONTIERS IN EDUCATION CONFERENCE (FIE), USA, 2016. Proceedings. Institute of Electrical and Electronics Engineers, 2016. SIRVINSKAS, L.P. Meio ambiente e cidadania. Revista do Instituto de Pesquisas e Estudos, Bauru, n.35, p. 305-307, 2002.

TAN, M.; When Makerspaces Meet School: Negotiating Tensions Between Instruction and Construction. Journal of Science Education and Technology, v.28, n.2, p 75-89, 2018.

TRUST, T.; MALOY, R.W.; EDWARDS, S. Learning through Making: Emerging and Expanding Designs for College Classes. Tech Trends, v.62, n.1, p 19-28, 2017.

UNESCO, Toward Universal Learning: What Every Child Should Learn, Center for Universal Education at Brookings Canada, 2013.

VARGAS, R. P.; HORTELANO, M. R.; ABAD, L. T.; CARRILlO, J.C; BERLINCHES, R.H. Teaching cloud computing using Web of Things devices. In: 2018 IEEE GLOBAL ENGINEERING EDUCATION CONFERENCE (EDUCON), Spain, 2018. Proceedings. Institute of Electrical and Electronics Engineers, 2018.

YEH, C-Y.; CHENG, Y-M.; HUANG, S.; LOU, S-J.; ZHOU, E. Constructing Competence Indicators of Makers for Information and Technology Education in Elementary School. In: 2018 3rd INTERNATIONAL CONFERENCE ON COMPUTER AND COMMUNICATION SYSTEMS (ICCCS), Japan, 2018. Proceedings. Institute of Electrical and Electronics Engineers, 2018.

YOUMANS, K.; VILLANUEVA, I. NADELSON, L.; BOUWMA, J.; LENZ, A.; LANCI, S. Makerspaces vs Engineering Shops: Initial Undergraduate Student Impressions. In: 2018 IEEE FRONTIERS IN EDUCATION CONFERENCE (FIE), USA, 2018. Proceedings. Institute of Electrical and Electronics Engineers, 2018.

ZHANG, S.; SONG, S.; CAO, P.; LIU, B.; WEI, S.; REN, R. The Research and Practice of Maker Education and Associated Industrial Development Trends in China. In: 2018 SEVENTH INTERNATIONAL CONFERENCE OF EDUCATION INNOVATION THROUGH TECHNOLOGY (EITT), New Zealand, 2018. Proceedings. Institute of Electrical and Electronics Engineers, 2018. 